\title{
Time-series expression profile analysis of fracture healing in young and old mice
}

\author{
CHUN YUAN and JINFANG CAI
}

\author{
Department of Orthopedics, Jinan Military General Hospital, Jinan, Shandong 250031, P.R. China
}

Received October 10, 2016; Accepted June 13, 2017

DOI: $10.3892 / \mathrm{mmr} .2017 .7198$

\begin{abstract}
Bone fracture healing is a complex process, which is associated with several factors, including age and osteoporosis. Certain genes and biological processes that may contribute to fracture healing, have been identified following developments in systems biology and molecular biology technologies, which may benefit the treatment of bone fractures. The present study identified key genes, which may be important in fracture healing through bioinformatics analysis of gene microarray datasets from the Gene Expression Omnibus. Gene clusters, which were consistently up/downregulated through time following a fracture in young (6-week-old) mice and old (8-month-old retired breeders) mice were obtained via soft clustering of differentially expressed genes (DEGs) between samples at 1 and 3 days, 1 and 5 days, and 3 and 5 days post-fracture in the two age groups, based on the Mfuzz package of $R$. Functional enrichment analysis of gene clusters using the Database for Annotation, Visualization and Integrated Discovery indicated that biological processes and pathways, including those associated with bone development, skeletal system development, amino sugar and nucleotide sugar metabolism, were significantly enriched in these up/downregulated genes. Of note, a total of 207 overlapped consistently upregulated genes were obtained between the two age groups, whereas no overlap was identified between the two lists of consistently downregulated genes. The overlapped genes were found to be associated with the biological processes of blood vessel development, vasculature development and skeletal system development, compared with all genes in the clusters. In addition, certain genes, including epidermal growth factor-like domain multiple 6 (EGFL6), kazal-type serine peptidase inhibitor domain 1 (KAZALD1), olfactomedin 2B (OLFM2B), collagen type III $\alpha 1$ (COL3A1),
\end{abstract}

Correspondence to: Dr Chun Yuan, Department of Orthopedics, Jinan Military General Hospital, 25 Shifan Road, Jinan, Shandong 250031, P.R. China

E-mail: yuanchunpostdoc@126.com

Key words: fracture healing, bioinformatics, Gene Expression Omnibus, reverse transcription-quantitative polymerase chain reaction collagen type II $\alpha 1(C O L 2 A 1)$, von Willebrand factor A domain-containing 1 (VWA1), elastin microfibril interfacer 1 (EMILIN1) and aggrecan $(A C A N)$, of the extracellular matrix organization, a process performed at the cellular level and resulting in the assembly and arrangement of constituent parts, were confirmed to be associated with fracture healing via reverse transcription-quantitative polymerase chain reaction analysis. The present study identified certain genes and biological processes/pathways, which may be associated with fracture healing and may assist in fundamental investigations and treatment in the future.

\section{Introduction}

Fracture healing is a complex process and the major challenges associated with fracture healing are aging and osteoporosis (1). At present, $\sim 40 \%$ of individuals worldwide aged $\geq 60$ years have osteoporosis, and almost $30 \%$ of these individuals are likely to suffer an osteoporotic fracture $(1,2)$. Due to the defective ability of fracture repair, the incidence of fracture nonunion or delayed union is high in older patients, resulting in elevated morbidity rates and high costs of care for patients.

It has been reported that certain pathological factors may also affect fracture healing. For example, with developments in molecular biology, an increasing number of genes and biological processes have been found to be associated with fracture healing, and the subsequent emergence of corresponding small bioactive molecules may assist in the treatment of fracture nonunion. Previous evidence suggests that Scx may be involved in the periosteal response during fracture healing (3). Fischerauer et al (4) demonstrated that bone morphogenetic protein receptor, type IA (BMPR1A) was upregulated in the growth plate of the fractured tibia, and Morgan et al (5) found that a BMPR1Aantagonist affected cartilage and bone formation during fracture healing. These findings indicate the importance of examining the mechanisms of fracture healing for its adjuvant treatment.

Soft clustering, also termed fuzzy clustering, was first described by Dunn in 1973 (6). Compared with a hard clustering method, including K-means clustering, the data points (in this case genes) can potentially belong to multiple clusters. In 2007, Kumar and Futschik developed Mfuzz (7), which is an $\mathrm{R}$ package specific for the soft clustering of gene microarray data, and Chen et al (8) identified certain target molecules for 
colorectal cancer through the analysis of time-series gene expression profiles based on Mfuzz.

In the present study, bioinformatics analyses, including differential expression analysis, soft clustering and functional enrichment analysis, were performed on microarray datasets from the Gene Expression Omnibus (GEO; https://www.ncbi .nlm.nih.gov/geo/) and this was followed by further confirmation of genes potentially contributing to fracture healing through reverse transcription-quantitative polymerase chain reaction (RT-qPCR) analysis. Certain genes, which have received less attention but are also important in fracture healing, were also included in the present study for evaluation. The majority of the enriched Gene Ontology (GO) terms and Kyoto Encyclopedia of Genes and Genomes (KEGG) pathways of these genes are associated with fracture healing. The findings of the present study may improve current understanding of fracture healing and provide potential therapeutic targets for further investigation.

\section{Materials and methods}

Microarray datasets. The microarray dataset (GSE17825) investigated in the present study was downloaded from the GEO (https://www.ncbi.nlm.nih.gov/geo/), which contained 18 femur samples from catagmatic female c57BI6 mice. Fracture calluses were dissected from old (8-month-old retired breeders) and young (6-week-old) mice at 1, 3 and 5 days post-fracture, and total RNA was isolated in triplicate for every group. The relative mRNA level was quantified using a GPL1261 [Mouse430_2] Affymetrix Mouse Genome 430 2.0 array and raw data were deposited by Khan et al (9).

Microarray data analysis. The raw microarray data were imported into R 3.22 and normalized using the Affy package version 1.54.0 (https://bioconductor.org/packages/release/bioc/html/affy.html) (10). The probe ID was transformed into gene symbols and the expression values for genes corresponding to multiple probes were summarized. Using the Limma package version 3.32.2 (https://bioconductor .org/packages/release/bioc/html/limma.html) (11), differentially expressed genes (DEGs) were identified between samples at 1 and 3 days, 1 and 5 days, and 3 and 5 days post-fracture in the old and young groups, according to the criteria of an adjusted $\mathrm{P}<0.05$ and fold change $>2$.

To identify genes consistently up/downregulated with time following fracture, a soft clustering method, which allows multi-assignment in different clusters for a specific gene according to how well the gene is represented by those clusters, was applied to the DEGs in the old and young groups, respectively, based on the Mfuzz package version 2.36.0 (https://www .bioconductor.org/packages/release/bioc/html/Mfuzz.html).

For this, the cluster number was set to 10 and a score of 0.9 was used for the determination of which clusters each DEG belonged to.

Functional enrichment analysis. Complex biological processes may be involved in fracture healing. For the consistently up/downregulated DEGs in the old and young groups obtained through clustering, significantly enriched $(\mathrm{P}<0.05)$ Gene Ontology (GO) terms and Kyoto Encyclopedia of
Genes and Genomes (KEGG) pathways were obtained using the Database for Annotation, Visualization and Integrated Discovery (http://david.abcc.ncifcrf.gov/) (12). In addition, the DEGs consistently up/downregulated with time following fracture in the old and young groups may indicate key genes in fracture healing, therefore, the enriched GO terms and KEGG pathways of these genes were also obtained.

Animals. Female C57BL/6 mice were purchased from Joint Ventures Sipper BK Experimental Animal Company (Shanghai, China), and mice weighing 30.0 $\pm 5.0 \mathrm{~g}$ were used in the present study. All animals were between 6 and 8 weeks of age at the initiation of the investigation. The animals were housed in specific pathogen-free conditions $\left(18-29^{\circ} \mathrm{C} ; 40-70 \%\right.$; free access to food and water) and experiments were approved by the Institutional Animal Use and Care Committee of Jinan Military General Hospital (Jinan, China).

RNA isolation. Mice were housed and maintained in the aforementioned conditions until they reached 8-12 weeks old, and then fractures were induced. Fractures were carefully opened through the same muscular interval as previously described (9). With careful anatomization, the same interval was distinguished and enrolled, and early hematoma and soft callous was maintained. The central third of the femur, including all callous or hematoma, was removed and flash frozen in liquid nitrogen. Tissues were prepared for RNA isolation as previously described (9). Total RNA from the cells were isolated with TRIzol reagent (Invitrogen; Thermo Fisher Scientific, Inc., Waltham, MA, USA) and treated with DNase I, followed by reverse transcription with random primers and AMV reverse transcription (Thermo Fisher Scientific, Inc.). The total RNA isolated was combined from three animals for each separate array time point.

qPCR analysis. A $1 \mu \mathrm{g}$ sample of the total RNA was used for cDNA synthesis. The mRNA levels of bone morphogenetic protein 1 (BMPl), collagen type XIII $\alpha 1$ (COL13A1), SP3, kazal-type serine peptidase-inhibitor domain 1 (KAZALDl), insulin-induced gene 1 (INSIGI), collagen type II $\alpha 1$ (COL2A1), matrix metalloproteinase-14 (MMP14) and 3'-phosphoadenosine 5'-phosphosulfate synthetase 2 (PAPSS2) were determined using RT-qPCR analysis with SYBR ${ }^{\circledR}$ Green Real-time PCR Master Mix (Thermo Fisher Scientific, Inc.). The following primers were used: BMP1, forward 5'-ACCCT CCAAGACAGC ACTG'-3 and reverse 5'-GGCTACGGTA CAGGTCCAT-3'; COL13A1, forward 5'-CGGACCTAAA GGGGAGATGG-3' and reverse 5'-CCAGCCGCACTGAAT TGAGT-3'; SP3, forward 5'-TCAAGTAGTCGCTAATGTGC CT-3' and reverse 5'-GAACTTCCCGAGAGTCCCAA A-3'; $K A Z A L D 1$, forward 5'-GCTTCTGCAACCCTCACAGT-3' and reverse 5'-TCAGGAAACAAACTCCGTGATTG-3'; INSIG1, forward 5'-TGTCGGTTTACTGTATCCCTGT-3' and reverse 5'-GTTGATGCCAACGAACACGG-3'; COL2A1, forward 5'-GGGTCACAGAGGTTACCCAG-3' and reverse 5'-ACCAGGGGAACCACTCTCAC-3'; MMP14, forward 5'-GCTTCCGAGAAGTGCCCTATG-3' and reverse, 5'-TCCTTCACCATCAAAGGGTGTA-3'; PAPSS2, forward 5'-GACCAGCAAAAATCCACCAATG-3' and reverse 5'-CACACGGTACATCCTCGGAAT-3'; GAPDH, forward 
Table I. Distribution of differentially expressed genes.

\begin{tabular}{lrc}
\hline Group & Downregulated (n) & Upregulated (n) \\
\hline Young & & \\
Day 1-3 & 130 & 223 \\
Day 1-5 & 470 & 1,171 \\
Day 3-5 & 4 & 51 \\
Old & & \\
Day 1-3 & 640 & 1,686 \\
Day 1-5 & 1,066 & 2,086 \\
Day 3-5 & 79 & 218 \\
\hline
\end{tabular}
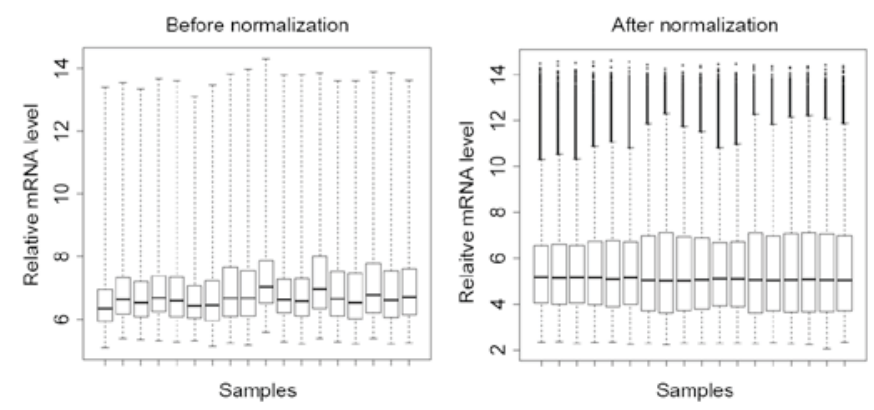

Figure 1. Boxplot of the relative mRNA levels in each sample prior to (left panel) and following (right panel) normalization. The $\mathrm{X}$-axis represents the samples used in the present study.
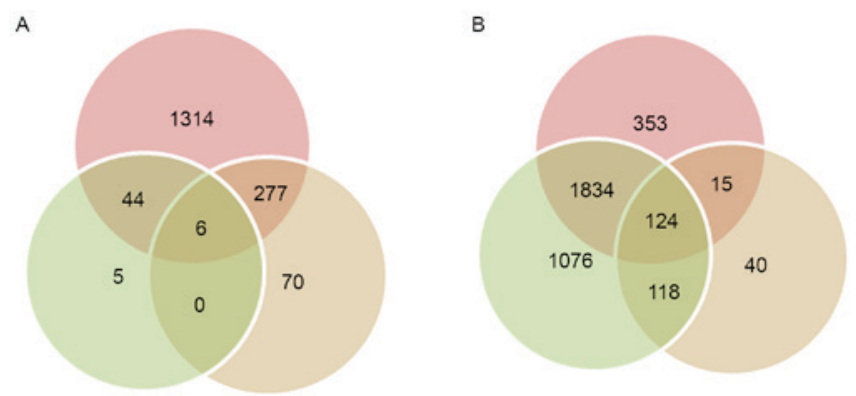

Figure 2. Venn diagram of overlapped genes among the differentially expressed genes between 1 and 3 days, 1 and 5 days, and 3 and 5 days in mice of the (A) young and (B) old groups.

5'-AGGTC GGTGT GAACG GATTT G and reverse 5'-GGGGT CGTTG ATGGC AACA-3'. The reactions were performed using an ABI 7,500 Fast Real-time PCR system. The thermocycling conditions were as follows: Initial denaturation step at $95^{\circ} \mathrm{C}$ for $10 \mathrm{~min}$, followed by 40 cycles at $95^{\circ} \mathrm{C}$ for $15 \mathrm{sec}, 60^{\circ} \mathrm{C}$ for $30 \mathrm{sec}$ and $72^{\circ} \mathrm{C}$ for $30 \mathrm{sec}$. The quantity of the gene was determined relative to the GAPDH quantification cycle using the $2^{-\Delta \Delta \mathrm{Cq}}$ method (13).

Statistical analysis. The relative mRNA levels are presented as the mean \pm standard deviation and were analyzed using $R$ programming software version 3.4.0 (https://cran.r-project. org/). $\mathrm{P}<0.05$ was considered to indicate a statistically significant difference, obtained using (two-tailed) Student's t-test.

\section{Results}

Microarray analysis. The relative mRNA levels in every sample prior to and following normalization are shown in Fig. 1. Comparable mRNA levels were obtained following normalization, compared with the raw data, and this was used for the following analysis. The distribution of DEGs between samples at 1 and 3 days, 1 and 5 days, and 3 and 5 days in the old and young groups are shown in Table I. A total of six and 124 overlaps were obtained among the three groups of DEGs for the young and old groups, respectively (Fig. 2).

For the DEGs consistently upregulated in young and old groups, several common GO terms were identified, including extracellular region part, bone development, and skeletal system development. By contrast, biological processes associated with the inflammatory response and protein binding were found to be enriched in the consistently downregulated DEGs the in old and young groups, respectively. As with the GO terms, a number of common KEGG pathways were identified for the DEGs consistently upregulated in the young and old groups, including Lysosome, Amino sugar and nucleotide sugar metabolism, and Focal adhesion. For the DEGs consistently downregulated in young and old groups, they were found to be involved in KEGG pathways, including Lysosome, Leukocyte transendothelial migration, and Cytokine-cytokine receptor interaction, and Cardiac muscle contraction, respectively. The top 10 significantly enriched GO terms and all KEGG pathways are listed in Tables II and III.

Soft clustering. According to the results obtained from the Mfuzz program, a total of four clusters (Fig. 3A; clusters 2, 3, 6 and 9) and one cluster (Fig. 3A; cluster 1) were found to be consistently upregulated and downregulated, respectively, in the young group. In the old group, a total of three clusters (Fig. 3B; cluster 1, 5 and 10) and one cluster (Fig. 3B; cluster 3) were consistently upregulated and downregulated, respectively. The number of consistently upregulated and downregulated DEGs in the young and old groups were 596 and 1,228, and 231 and 338, respectively. Of note, a total of 207 overlaps were obtained between the consistently upregulated DEGs in the young and old groups, whereas no overlap was identified between the consistently downregulated DEGs. In addition, the supervised clustering based on these 207 overlapped consistently upregulated DEGs separated all the samples, with the exception of the old and young samples at 5 days post-fracture, into their own groups according to the age or duration following fracture (Fig. 4). The top 10 significantly enriched GO terms and KEGG pathways of these 207 overlaps are listed in Table IV.

RT-qPCR validation of involvement of extracellular matrix (ECM) organization in fracture healing. ECM organization is a process, which occurs at the cellular level, and results in the assembly and arrangement of constituent parts, or the disassembly of ECM. Previous studies have noted the importance of ECM organization in several biological processes, including tumor cell migration and immune defense. The present study performed comprehensive analysis of the 207 overlapped genes consistently upregulated overtime following fracture in young and old groups, and found that 
Table II. Top 10 significantly enriched GO terms of genes in consistently upregulated and downregulated clusters.

\begin{tabular}{|c|c|c|}
\hline Category & GO term & P-value \\
\hline \multicolumn{3}{|c|}{ Upregulated in young } \\
\hline $\mathrm{CC}$ & Extracellular region part & $9.47 \times 10^{-12}$ \\
\hline $\mathrm{CC}$ & Extracellular region & $2.24 \times 10^{-9}$ \\
\hline $\mathrm{CC}$ & Extracellular matrix & $5.78 \times 10^{-9}$ \\
\hline $\mathrm{CC}$ & Proteinaceous extracellular matrix & $1.02 \times 10^{-8}$ \\
\hline $\mathrm{BP}$ & Bone development & $1.08 \times 10^{-6}$ \\
\hline $\mathrm{BP}$ & Skeletal system development & $2.33 \times 10^{-6}$ \\
\hline $\mathrm{BP}$ & Aminoglycan metabolic process & $1.12 \times 10^{-5}$ \\
\hline $\mathrm{BP}$ & Polysaccharide metabolic process & $2.16 \times 10^{-5}$ \\
\hline $\mathrm{BP}$ & Proteoglycan metabolic process & $5.45 \times 10^{-5}$ \\
\hline $\mathrm{BP}$ & Ossification & $7.39 \times 10^{-5}$ \\
\hline \multicolumn{3}{|c|}{ Downregulated in young } \\
\hline $\mathrm{CC}$ & Endoplasmic reticulum & $9.60 \times 10^{-7}$ \\
\hline MF & ER retention sequence binding & $3.47 \times 10^{-4}$ \\
\hline MF & Inorganic cation transmembrane transporter activity & $4.59 \times 10^{-4}$ \\
\hline $\mathrm{BP}$ & Vesicle-mediated transport & $7.40 \times 10^{-4}$ \\
\hline MF & Protein domain specific binding & $1.16 \times 10^{-3}$ \\
\hline $\mathrm{BP}$ & Protein retention in ER lumen & $1.22 \times 10^{-3}$ \\
\hline MF & Transition metal ion transmembrane transporter activity & $2.14 \times 10^{-3}$ \\
\hline MF & PDZ domain binding & $2.41 \times 10^{-3}$ \\
\hline MF & Signal sequence binding & $3.13 \times 10^{-3}$ \\
\hline MF & Enzyme binding & $3.48 \times 10^{-3}$ \\
\hline \multicolumn{3}{|c|}{ Upregulated in old } \\
\hline $\mathrm{CC}$ & Extracellular matrix & $2.24 \times 10^{-26}$ \\
\hline $\mathrm{CC}$ & Proteinaceous extracellular matrix & $2.24 \times 10^{-25}$ \\
\hline $\mathrm{CC}$ & Extracellular region part & $1.37 \times 10^{-18}$ \\
\hline $\mathrm{BP}$ & Cell adhesion & $1.75 \times 10^{-14}$ \\
\hline $\mathrm{BP}$ & Biological adhesion & $1.93 \times 10^{-14}$ \\
\hline $\mathrm{BP}$ & Extracellular matrix organization & $1.87 \times 10^{-13}$ \\
\hline $\mathrm{BP}$ & Skeletal system development & $2.23 \times 10^{-13}$ \\
\hline $\mathrm{BP}$ & Extracellular structure organization & $4.32 \times 10^{-13}$ \\
\hline $\mathrm{BP}$ & Bone development & $2.34 \times 10^{-12}$ \\
\hline $\mathrm{CC}$ & Extracellular region & $3.82 \times 10^{-11}$ \\
\hline \multicolumn{3}{|c|}{ Downregulated in old } \\
\hline $\mathrm{CC}$ & Myofibril & $2.23 \times 10^{-8}$ \\
\hline $\mathrm{CC}$ & Contractile fiber & $3.52 \times 10^{-8}$ \\
\hline MF & Chemokine activity & $6.11 \times 10^{-7}$ \\
\hline MF & Chemokine receptor binding & $7.37 \times 10^{-7}$ \\
\hline $\mathrm{CC}$ & Sarcomere & $8.26 \times 10^{-7}$ \\
\hline $\mathrm{CC}$ & Contractile fiber part & $1.53 \times 10^{-6}$ \\
\hline $\mathrm{CC}$ & I band & $4.83 \times 10^{-6}$ \\
\hline $\mathrm{BP}$ & Cellular ion homeostasis & $1.69 \times 10^{-4}$ \\
\hline $\mathrm{BP}$ & Cellular cation homeostasis & $1.75 \times 10^{-4}$ \\
\hline $\mathrm{BP}$ & Inflammatory response & $1.85 \times 10^{-4}$ \\
\hline
\end{tabular}

GO, Gene Ontology; CC, cellular component; MF, molecular function; BP, biological process.

ECM organization was significantly enriched. A total of eight genes were included in this GO term in the present study, namely EGFL6, KAZALD1, OLFML2B, COL3A1, ACAN,
COL2A1, VWA1 and EMILIN1. To confirm the important genes involved in the pathogenesis of fracture healing, femur tissues from 10 young mice at 1 day post-fracture and 
Table III. Top 10 significantly enriched KEGG pathways of genes in consistently upregulated and downregulated clusters.

\begin{tabular}{lll}
\hline KEGG pathway $\quad$ Genes (n) $\quad$ P-value
\end{tabular}

Genes upregulated in young

samples

Lysosome

Chondroitin sulfate biosynthesis

Amino sugar and nucleotide

sugar metabolism

Thyroid cancer

Chemokine signaling pathway

Glycosphingolipid biosynthesis

Other glycan degradation

Focal adhesion

Downregulated in young samples

\begin{tabular}{|c|c|c|}
\hline Lysosome & 6 & $1.22 \times 10^{-2}$ \\
\hline Cell adhesion molecules & 6 & $3.32 \times 10^{-2}$ \\
\hline $\begin{array}{l}\text { Leukocyte transendothelial } \\
\text { migration }\end{array}$ & 5 & $4.96 \times 10^{-2}$ \\
\hline \multicolumn{3}{|l|}{ Upregulated in old samples } \\
\hline Focal adhesion & 30 & $1.75 \times 10^{-7}$ \\
\hline ECM-receptor interaction & 17 & $3.13 \times 10^{-6}$ \\
\hline Colorectal cancer & 12 & $3.95 \times 10^{-3}$ \\
\hline Gap junction & 12 & $3.95 \times 10^{-3}$ \\
\hline Lysosome & 14 & $7.11 \times 10^{-3}$ \\
\hline $\mathrm{N}$-Glycan biosynthesis & 8 & $7.96 \times 10^{-3}$ \\
\hline TGF- $\beta$ signaling pathway & 11 & $1.23 \times 10^{-2}$ \\
\hline Adherens junction & 10 & $1.43 \times 10^{-2}$ \\
\hline $\begin{array}{l}\text { Amino sugar and nucleotide } \\
\text { sugar metabolism }\end{array}$ & 7 & $2.28 \times 10^{-2}$ \\
\hline Pathways in cancer & 25 & $3.81 \times 10^{-2}$ \\
\hline Prostate cancer & 10 & $3.85 \times 10^{-2}$ \\
\hline $\begin{array}{l}\text { Leukocyte transendothelial } \\
\text { migration }\end{array}$ & 12 & $3.89 \times 10^{-2}$ \\
\hline Tight junction & 13 & $4.13 \times 10^{-2}$ \\
\hline $\begin{array}{l}\text { SNARE interactions in vesicular } \\
\text { transport }\end{array}$ & 6 & $4.18 \times 10^{-2}$ \\
\hline $\begin{array}{l}\text { Biosynthesis of unsaturated } \\
\text { fatty acids }\end{array}$ & 5 & $4.58 \times 10^{-2}$ \\
\hline \multicolumn{3}{|l|}{ Downregulated in old samples } \\
\hline $\begin{array}{l}\text { Cytokine-cytokine receptor } \\
\text { interaction }\end{array}$ & 12 & $9.49 \times 10^{-4}$ \\
\hline Cardiac muscle contraction & 7 & $1.03 \times 10^{-3}$ \\
\hline Nitrogen metabolism & 4 & $4.64 \times 10^{-3}$ \\
\hline Hematopoietic cell lineage & 6 & $8.22 \times 10^{-3}$ \\
\hline Adipocytokine signaling pathway & 5 & $1.76 \times 10^{-2}$ \\
\hline Chemokine signaling pathway & 8 & $1.88 \times 10^{-2}$ \\
\hline Parkinson's disease & 6 & $4.86 \times 10^{-2}$ \\
\hline
\end{tabular}

KEGG, Kyoto Encyclopedia of Genes and Genomes.

young mice 5 days post-fracture were subjected to RT-qPCR analysis (Fig. 5).
Table IV. Top 10 significantly enriched GO terms and KEGG pathways of the 207 genes consistently upregulated with time following fracture in young and old groups.

\begin{tabular}{lrc}
\hline Term/pathway & Genes (n) & P-value \\
\hline GO term & & \\
Cell adhesion & 19 & $5.11 \times 10^{-6}$ \\
Biological adhesion & 19 & $5.24 \times 10^{-6}$ \\
Extracellular matrix & 8 & $4.61 \times 10^{-5}$ \\
organization & & \\
Extracellular structure & 9 & $8.16 \times 10^{-5}$ \\
organization & & \\
Blood vessel development & 11 & $1.03 \times 10^{-4}$ \\
Bone development & 8 & $1.24 \times 10^{-4}$ \\
Vasculature development & 11 & $1.26 \times 10^{-4}$ \\
Proteoglycan metabolic & 5 & $2.49 \times 10^{-4}$ \\
process & & \\
Angiogenesis & 8 & $2.60 \times 10^{-4}$ \\
Skeletal system development & 11 & $3.62 \times 10^{-4}$ \\
KEGG pathway & & \\
Focal adhesion & 7 & $5.16 \times 10^{-3}$ \\
Tight junction & 5 & $2.39 \times 10^{-2}$ \\
ECM-receptor interaction & 4 & $3.00 \times 10^{-2}$ \\
\hline
\end{tabular}

GO, Gene Ontology; KEGG, Kyoto Encyclopedia of Genes and Genomes.

\section{Discussion}

Despite considerable efforts to determine the mechanisms of fracture healing, current understanding of the genetic changes associated with the progression of fracture healing remains to be elucidated. In the present study, genome-wide gene expression analysis was performed using a high throughput method to identify the DEGs from samples 1, 3 and 5 days following fracture in 8-month-old retired breeding mice and 6 -week-old mice. Soft clustering identified consistently up/downregulated genes in the young and old groups over time following fracture, which indicate their possible involvement in fracture healing. In addition, 207 genes were identified as being consistently upregulated in the young and old mice, providing valuable targets for future investigations.

The $\mathrm{GO}$ analyses revealed that the significant $\mathrm{GO}$ categories included cell adhesion, ECM organization, blood vessel development, and bone development. The replacement of cartilage by bone during endochondral ossification is essential in fetal skeletal development, bone growth and fracture healing. The regulation of this process by the ECM occurs through matrix remodeling, involving a variety of cell attachment molecules and growth factors, which affect cell morphology and protein expression. The present study identified eight genes, including EGFL6, KAZALD1, OLFML2B, COL3A1, ACAN, COL2A1, $V W A 1$ and EMILIN1, which were associated with cell adhesion and ECM organization. EGFL6 is a member of the EGF repeat superfamily, which are characterized by the presence 

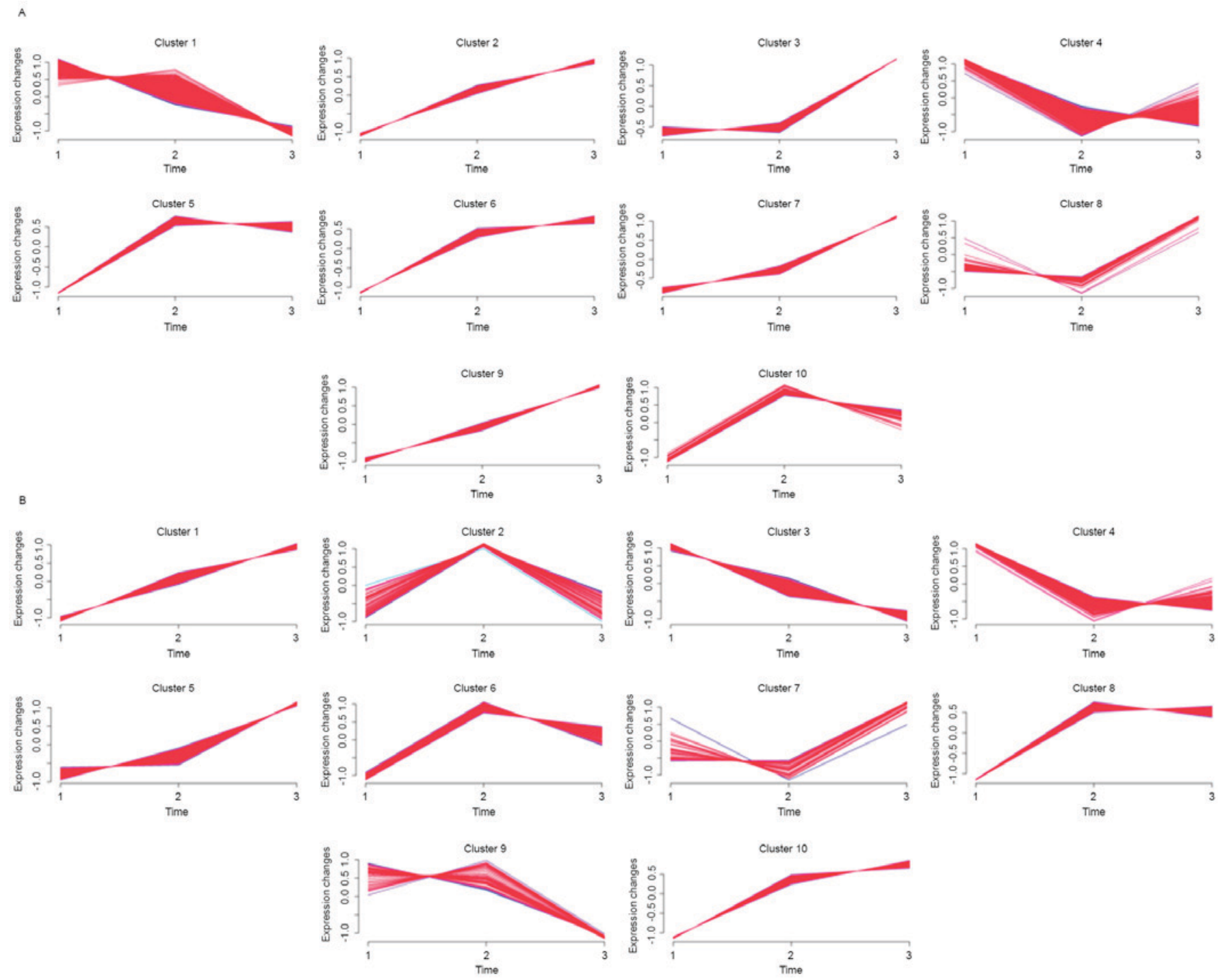

Figure 3. Clusters obtained via the soft clustering method for differentially expressed genes of mice in the (A) young and (B) old groups.

of EGF-like repeats, and are often involved in the regulation of cell cycle, proliferation and developmental processes. Chim et al (14) reported that EGLF6 mediates a paracrine mechanism of cross-talk between vascular endothelial cells and osteoblasts, and may offer an important novel target for the potential treatment of bone diseases, including osteonecrosis, osteoporosis and fracture healing. COL2Al is essential for normal embryonic development of the skeleton, for linear growth and for the ability of cartilage to resist compressive forces. In a previous study involving Col2a1-ICAT transgenic mice, radiological, histological and histomorphometric analyses 7, 9, 12, 14, 21 and 28 days following fracture demonstrated the bone repair was inhibited due to reduced and delayed cartilage formation, chondrocyte hypertrophy and bone generation (15). However, the remaining six genes have not been examined in reference to fracture healing, and the present study hypothesized that these genes may be critical markers, mediators or participants in the pathogenesis of fracture healing.

The 207 genes were then used in KEGG pathway analysis, which screened out four pathways, including Focal adhesion, Tight junction, ECM-receptor interaction, and Lysosome.
Previous studies have reported that the majority of these pathways are involved in fracture healing (16-18). However, in reviewing previous literature, there are no reports on the association between lysosome and fracture healing. Lysosomes are spherical vesicles, which contain hydrolytic enzymes that can break down almost all types of biomolecule (19). Therefore, the function of lysosomes in fracture healing warrants further investigation.

Taken together, through the identification of DEGs and performing clustering, GO enrichment, KEGG pathway analysis and RT-qPCR analysis, several key genes were screened out, which may be important in fracture healing, and a number of these genes were further confirmed. However, the results of the present study require further investigation to determine their potential utilization in the treatment of fractures.

\section{Acknowledgements}

The present study was supported by the General Financial Grant from the China Postdoctoral Science Foundation (grant no. 2013M532178). 

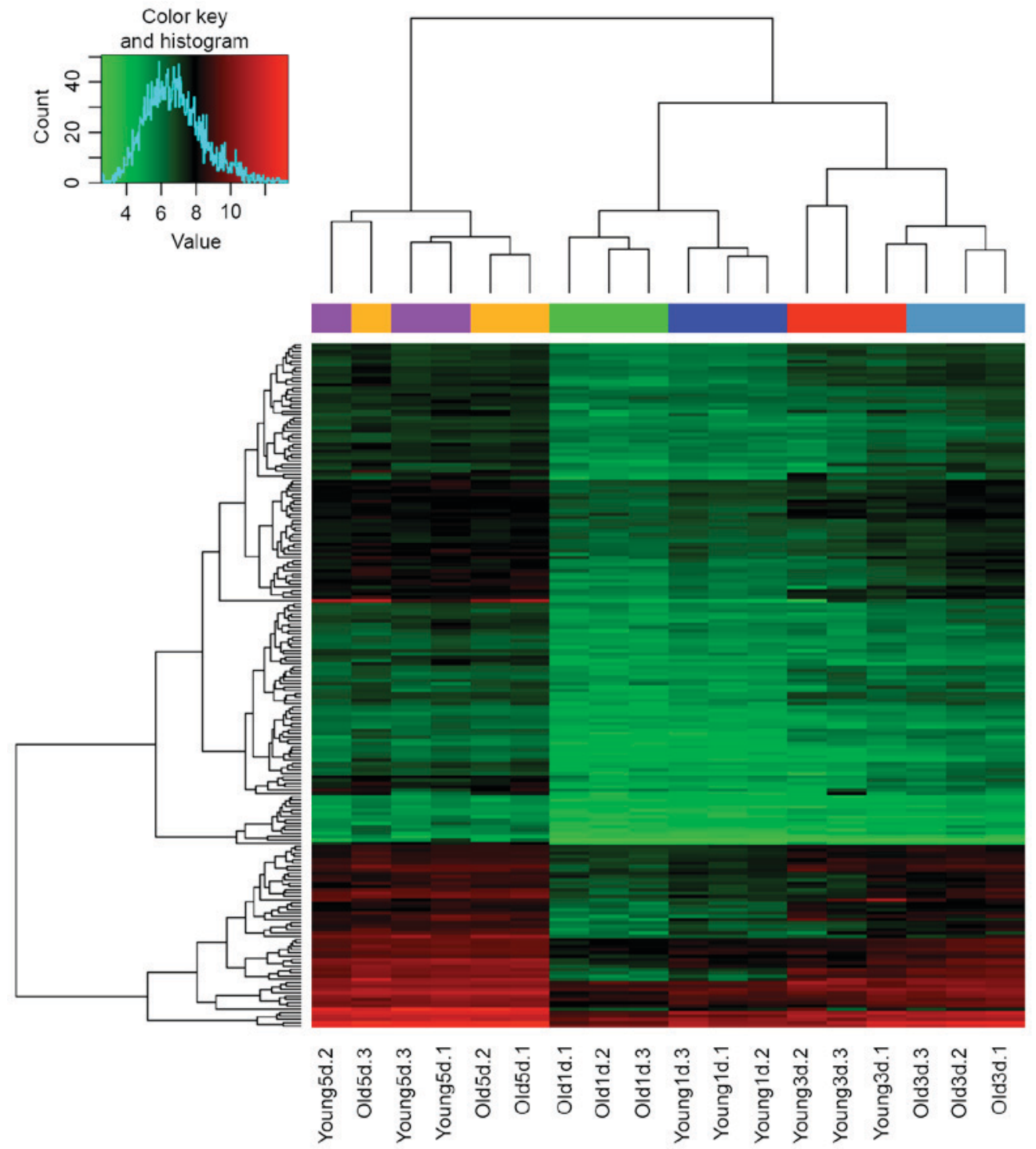

Figure 4. Supervised clustering of samples. A total of 207 genes were consistently upregulated in the young and old groups overtime following fracture. The green and red colors represent low and high expression values, respectively. The trees shown at the top and on the left, indicate sample and gene clusters, respectively. $1 \mathrm{~d}, 1$ day post-fracture; $3 \mathrm{~d}, 3$ days post-fracture; $5 \mathrm{~d}, 5$ days post-fracture.
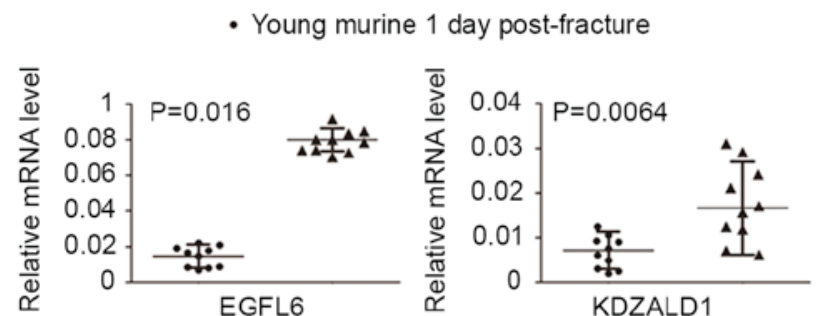

^ Young murine 5 days post-fracture
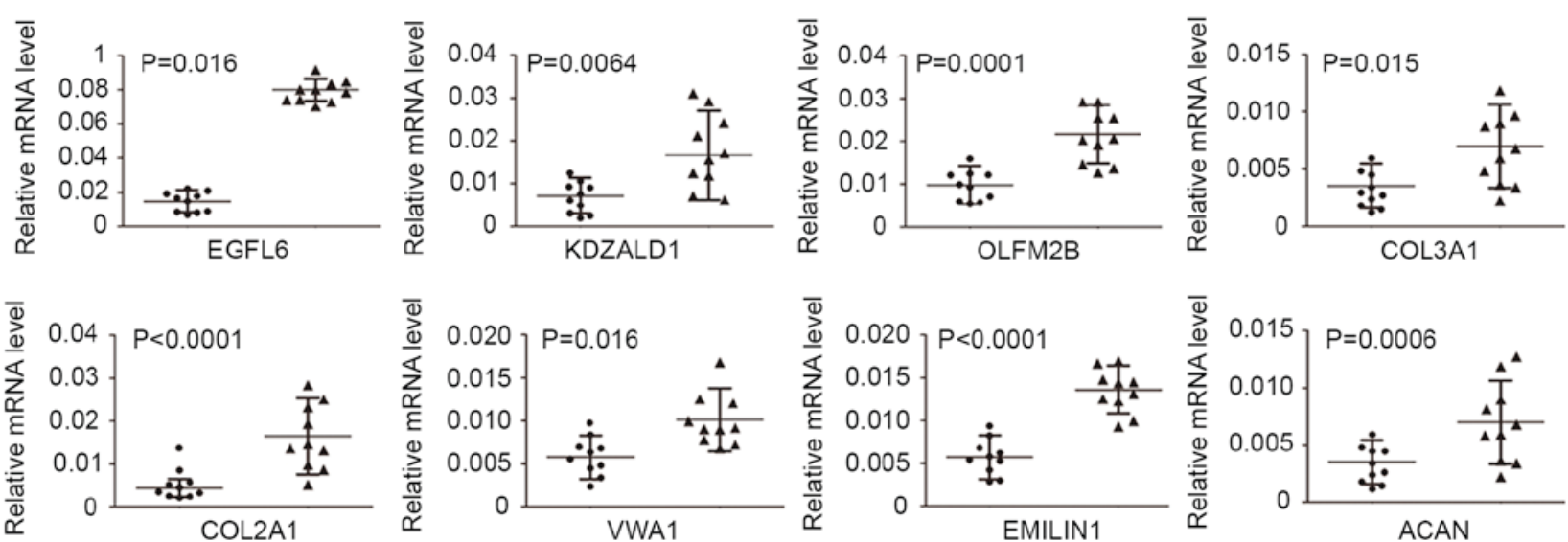

Figure 5. Relative expression level of genes included in the GO term of extracellular matrix organization. Expression levels were determined using reverse transcription-quantitative polymerase chain reaction in young mice at 1 and 5 days post-fracture. Data are expressed as the mean \pm standard deviation of three independent experiments ( ${ }^{*} \mathrm{P}<0.05$ and ${ }^{* *} \mathrm{P}<0.01$; two-tailed unpaired t-test). EGFL6, epidermal growth factor-like domain multiple 6; KAZALDI, kazal-type serine peptidase inhibitor domain 1; COL3A1, collagen type III $\alpha 1$; COL2A1, collagen type II $\alpha 1$, VWA1, von Willebrand factor A domain-containing 1, EMILIN1, elastin microfibril interfacer 1; ACAN, aggrecan. 


\section{References}

1. Foulke BA, Kendal AR, Murray DW and Pandit H: Fracture healing in the elderly: A review. Maturitas 92: 49-55, 2016.

2. Lamster IB, Asadourian L, Del Carmen T and Friedman PK: The aging mouth: Differentiating normal aging from disease. Periodontol 72: 96-107, 2016.

3. McKenzie JA, Buettmann E, Abraham AC, Gardner MJ, Silva MJ and Killian ML: Loss of scleraxis in mice leads to geometric and structural changes in cortical bone, as well as asymmetry in fracture healing. FASEB J 31: 882-892, 2017.

4. Fischerauer EE, Manninger M, Seles M, Janezic G, Pichler K, Ebner B and Weinberg AM: BMP-6 and BMPR-1a are up-regulated in the growth plate of the fractured tibia. J Orthop Res 31: 357-363, 2013 .

5. Morgan EF, Pittman J, DeGiacomo A, Cusher D, de Bakker CM, Mroszczyk KA, Grinstaff MW and Gerstenfeld LC: BMPR1A antagonist differentially affects cartilage and bone formation during fracture healing. J Orthop Res 34: 2096-2105, 2016.

6. Dunn JC: A fuzzy relative of the ISODATA process and its use in detecting compact well-separated clusters. Journal of Cybernetics 3: 32-57, 1973.

7. Kumar L and E Futschik M: Mfuzz: A software package for soft clustering of microarray data. Bioinformation 2: 5-7, 2007.

8. Chen G, Han N, Li G, Li X, Li G, Li Z and Li Q: Time course analysis based on gene expression profile and identification of target molecules for colorectal cancer. Cancer Cell Int 16: 22, 2016.

9. Khan SN, Solaris J, Ramsey KE, Yang X, Bostrom MP Stephan D and Daluiski A: Identification of novel gene expression in healing fracture callus tissue by DNA microarray. HSS J 4: 149-160, 2008.

10. Gautier L, Cope L, Bolstad BM and Irizarry RA: affy-analysis of Affymetrix GeneChip data at the probe level. Bioinformatics 20 : 307-315, 2004.
11. Diboun I, Wernisch L, Orengo CA and Koltzenburg M: Microarray analysis after RNA amplification can detect pronounced differences in gene expression using limma. BMC Genomics 7: 252, 2006

12. Dennis G Jr, Sherman BT, Hosack DA, Yang J, Gao W, Lane HC and Lempicki RA: DAVID: Database for annotation, visualization, and integrated discovery. Genome Biol 4: P3, 2003.

13. Livak KJ and Schmittgen TD: Analysis of relative gene expression data using real-time quantitative PCR and the 2(-Delta Delta C(T)) method. Methods 25: 402-408, 2001.

14. Chim SM, Qin A, Tickner J, Pavlos N, Davey T, Wang H, Guo Y, Zheng $\mathrm{MH}$ and $\mathrm{Xu}$ J: EGFL6 promotes endothelial cell migration and angiogenesis through the activation of extracellular signal-regulated kinase. J Biol Chem 286: 22035-22046, 2011.

15. Huang Y, Zhang X, Du K, Yang F, Shi Y, Huang J, Tang T, Chen $\mathrm{D}$ and Dai K: Inhibition of $\beta$-catenin signaling in chondrocytes induces delayed fracture healing in mice. J Orthop Res 30: 304-310, 2012.

16. Horikiri Y, Shimo T, Kurio N, Okui T, Matsumoto K, Iwamoto M and Sasaki A: Sonic hedgehog regulates osteoblast function by focal adhesion kinase signaling in the process of fracture healing. PloS One 8: e76785, 2013

17. Wang W, Shen H, Xie J,Zhou Q, Chen Y and Lu H: Bioinformatics analysis of time-series genes profiling to explore key genes affected by age in fracture healing. Mol Biol Rep 41: 3881-3889, 2014.

18. Jia Z, Zhang Y, Chen YH, Dusad A, Yuan H, Ren K, Li F, Fehringer EV, Purdue PE, Goldring SR, et al: Simvastatin prodrug micelles target fracture and improve healing. J Control Release 200: 23-34, 2015.

19. Kuech EM, Brogden G and Naim HY: Alterations in membrane trafficking and pathophysiological implications in lysosomal storage disorders. Biochimie 130: 152-162, 2016. 